\title{
Effects of dexmedetomidine on immune function, renal function and inflammatory factors of patients undergoing percutaneous nephrolithotomy under general anesthesia
}

\author{
YUYAN BAI, HEFAN HE, PEISEN ZHANG, WEIFENG LIU and LIRONG HUANG \\ Department of Anesthesiology, The Second Affiliated Hospital of Fujian \\ Medical University, Quanzhou, Fujian 362000, P.R. China
}

Received July 12, 2019; Accepted March 10, 2020

DOI: $10.3892 /$ etm.2021.9837

\begin{abstract}
The present study was designed to determine the effects of dexmedetomidine on immune function, renal function and inflammatory factors in patients undergoing percutaneous nephrolithotomy under general anesthesia. A total of 177 patients with kidney calculi who underwent percutaneous nephrolithotomy in The Second Affiliated Hospital of Fujian Medical University were enrolled, in which 91 patients were treated with dexmedetomidine during surgery (research group) and 86 patients were not sedated during surgery (control group). The vital signs, renal function, inflammatory factors and immune function during surgery between the two groups were compared. Patients in the research group showed improved vital signs, renal function, inflammatory factors and immune function compared with the control group $(\mathrm{P}<0.05)$, and also experienced a significantly shorter hospitalization time $(\mathrm{P}<0.001)$. Therefore, the present results suggested that with a relatively high safety profile, use of dexmedetomidine for sedation can effectively protect renal and immune functions, and reduce the inflammatory response of patients during percutaneous nephrolithotomy. Thus, dexmedetomidine may have be potentially applied in clinical practice.
\end{abstract}

\section{Introduction}

Calculi in the urinary system are an extremely common form of calculus in human body, which seriously affects the health of the patient (1). The incidence rate of calculus in the urinary system is $1-5 \%$ (2), and the most common forms are kidney calculi (3). Calculi are caused by abnormal aggregation of crystalline substances such as calcium, oxalic acid and uric

Correspondence to: Dr Hefan He, Department of Anesthesiology, The Second Affiliated Hospital of Fujian Medical University, 34 Zhongshan North Road, Quanzhou, Fujian 362000, P.R. China E-mail: hhfan1969@163.com

Key words: dexmedetomidine, percutaneous nephrolithotomy, kidney calculi, diastolic blood pressure, $\mathrm{CD}^{+}$ acid (4). Furthermore, kidney calculi are common amongst young and middle-aged men (5), and the incidence of kidney calculi is rapidly increasing (6). Kidney calculi, a very common lithiasis, are usually accompanied by varying degrees of flank soreness and pain, which seriously affect everyday life and mobility (7). Kidney calculi are caused by numerous factors, including age, sex, heredity, environment, diet and occupation, and internal body influencing factors including abnormal metabolism, denutrition and urinary tract infection $(8,9)$. Currently, kidney calculi are primarily treated by surgery and with further development of medical technology, percutaneous nephrolithotomy has become the first choice for the treatment of kidney calculi (10).

Percutaneous nephrolithotomy is a minimally invasive surgery associated with small trauma, a fast recovery effect and few postoperative complications, and its application value has been proven clinically. However, there are some limitations to the use of percutaneous nephrolithotomy in clinical practice, such as damage of the renal function of patients $(10,11)$. Percutaneous nephrolithotomy may increase the intrapelvic pressure of patients, resulting in urine reflux in the renal pelvis, thereby damaging the patients' renal function $(11,12)$. Therefore, it is important to choose a narcotic analgesic drug that has the ability to protect renal function. Moreover, in order to effectively lower the degree of renal injury caused to patients during surgery, anesthetics with protective effects on renal function are required in clinical practice. Dexmedetomidine is a $\alpha_{2}$ adrenoceptor agonist that not only has a protective effect on kidney tissues, cells and functions, but also has significant anti-inflammatory effects (13). Currently, there are limited studies on the application of dexmedetomidine in percutaneous nephrolithotomy. In order to determine the application value of dexmedetomidine, the present study enrolled patients undergoing kidney calculi surgery in The Second Affiliated Hospital of Fujian Medical University. The present study investigated the effects of dexmedetomidine on the renal function, inflammatory response and immune function of patients undergoing percutaneous nephrolithotomy. Thus, the present results may provide an accurate and reliable reference for future clinical selection of anesthetics for percutaneous nephrolithotomy. 


\section{Materials and methods}

Patient data. A total of 177 patients with renal calculi who underwent percutaneous nephrolithotomy in the second affiliated Hospital of Fujian Medical University (Quanzhou, China) from October 2016 to October 2019 were enrolled. Patient characteristics are shown in Table I. Overall, 91 patients were treated with dexmedetomidine during surgery (research group) and 86 patients were not sedated during surgery (control group). The present study has been approved by the Ethics Committee of The Second Affiliated Hospital of Fujian Medical University and all patients or their direct families signed an informed consent form.

Inclusion and exclusion criteria. Inclusion criteria were as follows: i) Patients with clinical manifestations in line with those of kidney calculi (14); ii) patients diagnosed with kidney calculi based on a series of examinations at The Second Affiliated Hospital of Fujian Medical University; iii) patients in line with indications of percutaneous nephrolithotomy and who underwent surgery in this hospital; iv) patients in grade I-II of the American Society of Anesthesiologists (ASA) classification (15); v) patients with complete case information; and vi) patients who were willing to cooperate with the experiment. Exclusion criteria were as follows: i) Patients with severe urinary tract obstructive diseases during surgery; ii) patients treated with a replaced surgery plan; iii) patients with tumors, other cardio-cerebrovascular diseases, other autoimmune diseases or neurological disorders; iv) patients allergic to some drugs; v) patients with organ failure, or hepatic and kidney function obstacle; vi) patients with a long-term history of taking sedative drugs; and vii) patients transferred to another hospital.

Methods. All patients were treated by percutaneous nephrolithotomy by senior surgeons from The Second Affiliated Hospital of Fujian Medical University. The patients were injected intramuscularly with $0.1 \mathrm{~g}$ of phenobarbital and $0.5 \mathrm{mg}$ of atropine before anesthesia. Patients were closely monitored in terms of vital signs after entering the operating room and peripheral venous access was established. After local anesthesia (intramuscular injection of $0.1 \mathrm{~g}$ of phenobarbital and $0.5 \mathrm{mg}$ of atropine), radial artery cannulation was performed and invasive arterial blood pressure was monitored. Patients in the research group were injected intravenously with $1 \mu \mathrm{g} / \mathrm{kg}$ dexmedetomidine (Jiangsu Heng Rui Pharmaceutical Co., Ltd.; State Food and Drug Administration approval no. H20090248) for $10 \mathrm{~min}$ prior to anesthesia induction and were then injected intravenously with dexmedetomidine at $0.5 \mu \mathrm{g} / \mathrm{kg} / \mathrm{h}$ (16) until the end of the surgery. The control group was given an equal amount of normal saline. Patients in the two groups were injected intravenously with etomidate $(0.3 \mathrm{mg}-\mathrm{kg})$, fentanyl $(0.3 \mu \mathrm{g} /$ $\mathrm{kg})$, midazolam $(0.1 \mathrm{mg} / \mathrm{kg})$ and atracurium $(0.15 \mathrm{mg}-\mathrm{kg})$ for anesthesia induction, and oral intubation was used for mechanical ventilation. The tidal volume was set to $8-10 \mathrm{ml} /$ $\mathrm{kg}$ and respiratory rate to $11-13$ times/min. The patients in the two groups were injected intravenously with propofol at $3-5 \mathrm{mg} / \mathrm{kg} / \mathrm{min}$ and remifentanil at 0.1-0.2 $\mu \mathrm{g}-\mathrm{kg}-\mathrm{min}$ for maintenance of anesthesia.
Observation indexes. Changes in vital signs, including diastolic blood pressure (DBP), systolic blood pressure (SBP), heart rate (HR) and blood oxygen saturation $\left(\mathrm{SpO}_{2}\right)$, were recorded before surgery (T0), 5 min after surgery (T1), 30 min after surgery (T2) and at the end of surgery (T3). The anesthesia wake-up time of the patient, which is the time from the end time point of surgery to the time point when the patient fully restored mental activity and could make sample movements (17), was recorded. The assessment of state of the patient at $24 \mathrm{~h}$ after surgery was based on the visual analogue scale (VAS) and Ramsay sedation scale $(18,19)$. Furthermore, the incidence of adverse reactions including nausea, vomiting, arrhythmia and hypotension were calculated as follows: The incidence of adverse reactions $=$ the number of patients with adverse reaction/the total number of patients $\mathrm{x} 100 \%$. Fasting venous blood $(4 \mathrm{ml})$ was sampled from the patients before surgery, 1 day after surgery and 3 days after surgery, and divided into two parts. One part was centrifuged for $10 \mathrm{~min}$ $\left(1,505 \times \mathrm{g}, 4^{\circ} \mathrm{C}\right)$ to obtain serum. Blood samples were analyzed using an automatic biochemistry analyzer for renal function determination, including blood urea nitrogen (BUN), serum creatinine (Scr), retinol binding protein (RBP) and cystatin C (Cys-C). Then, ELISA kits were used to determine tumor necrosis factor- $\alpha$ (TNF- $\alpha$; Xiamen Huijia Biotechnology Co., Ltd.; cat. no. orb-EHJ130636), interleukin-6 (IL-6; Shanghai Xin Yu Biotech Co.,Ltd.; cat. no. Bsk00040) and IL-8 (Shanghai Xiyuan Biotechnology Co., Ltd.; cat. no. XY-70R-35386) in the blood samples. Flow cytometry (BD canto II; BD Biosciences; Becton, Dickinson and Company) was used to detect the $\mathrm{T}$ lymphocyte subsets of patients, including the ratios of $\mathrm{CD}^{+}, \mathrm{CD}^{+}, \mathrm{CD}^{+}, \mathrm{CD} 4^{+} / \mathrm{CD} 8^{+}$and natural killer (NK) cells in another part of the blood samples, and the supporting CytExpert analysis software of the instrument (CytExpert v2.0; Beckman Coulter, Inc.) was adopted for analysis (20). The hospitalization time and hospitalization expense were compared between the two experimental groups.

Statistical analysis. All assays in the present study were repeated three times. Data were analyzed using SPSS 24.0 statistical software (Beijing Strong-Vinda Information Technology Co., Ltd.). All graphs were drawn using GraphPad 8 (Shenzhen SOFTHEAD Software Technology Co., Ltd.). Enumeration data, such as sex and incidence of adverse reactions, were presented as the rate, and comparison between these groups was analyzed using a $\chi^{2}$ test. Measurement data, such as DBP and HR, were expressed as the mean $\pm \mathrm{SD}$, and compared between groups using unpaired Student's t-test. Comparison of data before and after treatment was carried out using paired Student's t-test. In addition, comparison in measurement data at different time points was analyzed using a repeated measures ANOVA and Bonferroni post hoc test. $\mathrm{P}<0.05$ was considered to indicate a statistically significant difference.

\section{Results}

Comparison of patient data. There was no significant difference between the two groups in age, body mass index, operation time, intraoperative blood loss, sex, residential environment, smoking, exercise habits, nationality, ASA classification and calculi sites (Table I). 
Table I. Comparison of patient data.

\begin{tabular}{|c|c|c|c|c|}
\hline & Research group $(n=91)$ & Control group $(\mathrm{n}=86)$ & t or $c^{2}$ & P-value \\
\hline Age, year & $48.21 \pm 5.12$ & $49.13 \pm 5.29$ & 1.176 & 0.241 \\
\hline $\mathrm{BMI}, \mathrm{kg} / \mathrm{cm}^{2}$ & $25.62 \pm 2.26$ & $25.73 \pm 2.44$ & 0.311 & 0.756 \\
\hline Operation time, $\min$ & $124.24 \pm 8.62$ & $125.36 \pm 9.08$ & 0.842 & 0.401 \\
\hline Intraoperative blood loss, $\mathrm{ml}$ & $50.62 \pm 8.62$ & $51.12 \pm 8.76$ & 0.383 & 0.702 \\
\hline Sex & & & 0.337 & 0.561 \\
\hline Male & $79(86.81)$ & $72(83.72)$ & & \\
\hline Female & $12(13.19)$ & $14(16.28)$ & & \\
\hline Residential environment & & & 0.724 & 0.395 \\
\hline Urban area & $58(63.74)$ & $60(69.77)$ & & \\
\hline Rural area & $33(36.26)$ & $26(30.23)$ & & \\
\hline Smoking & & & 1.187 & 0.276 \\
\hline Yes & $72(79.12)$ & $62(72.09)$ & & \\
\hline No & $19(20.88)$ & $24(27.91)$ & & \\
\hline Exercise habit & & & 0.619 & 0.431 \\
\hline Yes & $12(13.19)$ & $15(17.44)$ & & \\
\hline None & $79(86.81)$ & $71(82.56)$ & & \\
\hline Nationality & & & 1.683 & 0.195 \\
\hline Han nationality & $87(95.60)$ & $85(98.84)$ & & \\
\hline Minority nationality & $4(4.40)$ & $1(1.16)$ & & \\
\hline ASA classification & & & 0.455 & 0.500 \\
\hline Grade I & $43(47.25)$ & $45(52.33)$ & & \\
\hline Grade II & $48(52.75)$ & $41(47.67)$ & & \\
\hline Calculi site & & & 0.060 & 0.806 \\
\hline Left kidney & $47(51.65)$ & $46(53.49)$ & & \\
\hline Right kidney & $44(48.35)$ & $40(46.51)$ & & \\
\hline
\end{tabular}

Data are presented as the mean \pm SD or $\mathrm{n}(\%)$. ASA, American Society of Anesthesiologists; BMI, body mass index.

Table II. Comparison between the two groups in adverse reactions.

\begin{tabular}{lcccc}
\hline & $\begin{array}{c}\text { Research group } \\
(\mathrm{n}=91)\end{array}$ & $\begin{array}{c}\text { Control group } \\
(\mathrm{n}=86)\end{array}$ & $\mathrm{c}^{2}$ & P-value \\
\hline Nausea & $1(1.10)$ & $1(1.16)$ & 0.002 & 0.968 \\
Vomiting & $2(2.20)$ & $2(2.33)$ & 0.003 & 0.954 \\
Arrhythmia & $0(0.00)$ & $1(1.16)$ & 1.064 & 0.302 \\
Hypotension & $2(2.20)$ & $3(3.49)$ & 0.268 & 0.605 \\
Diarrhea & $2(2.20)$ & $3(3.49)$ & 0.268 & 0.605 \\
Coma & $0(0.00)$ & $2(2.33)$ & 2.140 & 0.144 \\
Incidence, \% & 7.69 & 13.95 & 1.809 & 0.179 \\
\hline
\end{tabular}

Data are presented as the $\mathrm{n}(\%)$.

Comparison of vital signs. The two study groups showed no significant differences in SBP, DBP, $\mathrm{HR}$ and $\mathrm{SpO}_{2}$ at T0. It was demonstrated that the research group had lower SBP, DBP and HR compared with the control group at T1, T2 and T3 $(\mathrm{P}<0.05$; Fig. 1). Furthermore, the two groups showed lower SBP, DBP and HR at T1 compared with T0 $(\mathrm{P}<0.05)$, and had lower SBP, DBP and HR at T2 compared with both T0 and $\mathrm{T} 1(\mathrm{P}<0.05)$. In addition, at $\mathrm{T} 3$, the two groups had the lowest level of SBP $(\mathrm{P}<0.05)$, showed no difference in DBP compared with T2, had a higher HR compared with T1 and T2 and had a lower HR compared with T0 ( $<<0.001$; Fig. 1).

Comparisons of VAS score and Ramsay sedation scale score. The VAS score, Ramsay sedation scale score and anesthesia wake-up time of the research group were $0.57 \pm 0.26$ points, $4.35 \pm 0.25$ points and $216.22 \pm 16.27 \mathrm{~min}$, respectively (Fig. 2). Moreover, those of the control group were $1.09 \pm 0.19$ points, $2.42 \pm 0.32$ points and $168.67 \pm 16.08 \mathrm{~min}$, respectively. Thus, the present results suggested that the research group had a significantly lower VAS score and Ramsay sedation scale score compared with the control group ( $\mathrm{P}<0.001$; Fig. 2), and experienced a significantly longer anesthesia wake-up time compared with the control group $(\mathrm{P}<0.001)$.

Comparison of adverse reactions. The research group showed an incidence of adverse reactions of $7.69 \%$, with nausea in one patient $(1.10 \%)$, vomiting in two patients $(2.20 \%)$, hypotension in two patients $(2.20 \%)$ and diarrhea in two patients $(2.20 \%$; Table II). The control group had an incidence of adverse 

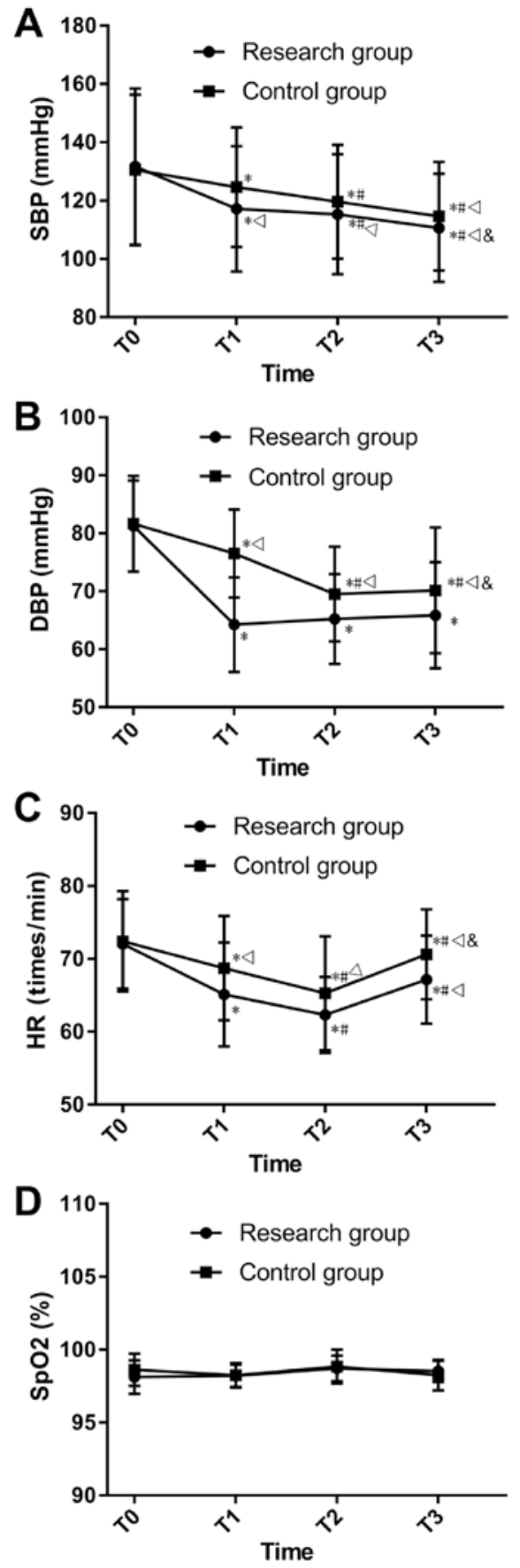

Figure 1. Comparison of vital signs. (A) Comparison of SBP. (B) Comparison of DBP. (C) Comparison of HR. (D) Comparison of $\mathrm{SpO}_{2}$. $\mathrm{P}<0.05$ vs. the same group at $\mathrm{T} 0 ;{ }^{\sharp} \mathrm{P}<0.001$ vs. the same group at $\mathrm{T} 1 ;{ }^{\triangleleft} \mathrm{P}<0.001$ vs. the same group at $\mathrm{T} 2 ;{ }^{\circledR} \mathrm{P}<0.001$ vs. the research group at the same time point. $\mathrm{T} 0$, before the surgery; T1, $5 \mathrm{~min}$ after the start of surgery; T2, 30 min after the start of surgery; T3, at the end of the surgery; SBP, systolic blood pressure DBP, diastolic blood pressure; $\mathrm{HR}$, heart rate; $\mathrm{SpO}_{2}$, blood oxygen saturation

reactions of $13.95 \%$, with nausea in one patient $(1.16 \%)$, vomiting in two patients $(2.33 \%)$, arrhythmia in one patient $(1.16 \%)$, hypotension in three patients $(3.49 \%)$, diarrhea in three patients $(3.49 \%)$ and coma in two patients $(2.33 \%)$. The two groups showed no significant difference in incidence of adverse reactions (Table II).

Comparison of renal function. The two groups showed no significant difference in BUN, RBP and Cys-C before surgery,

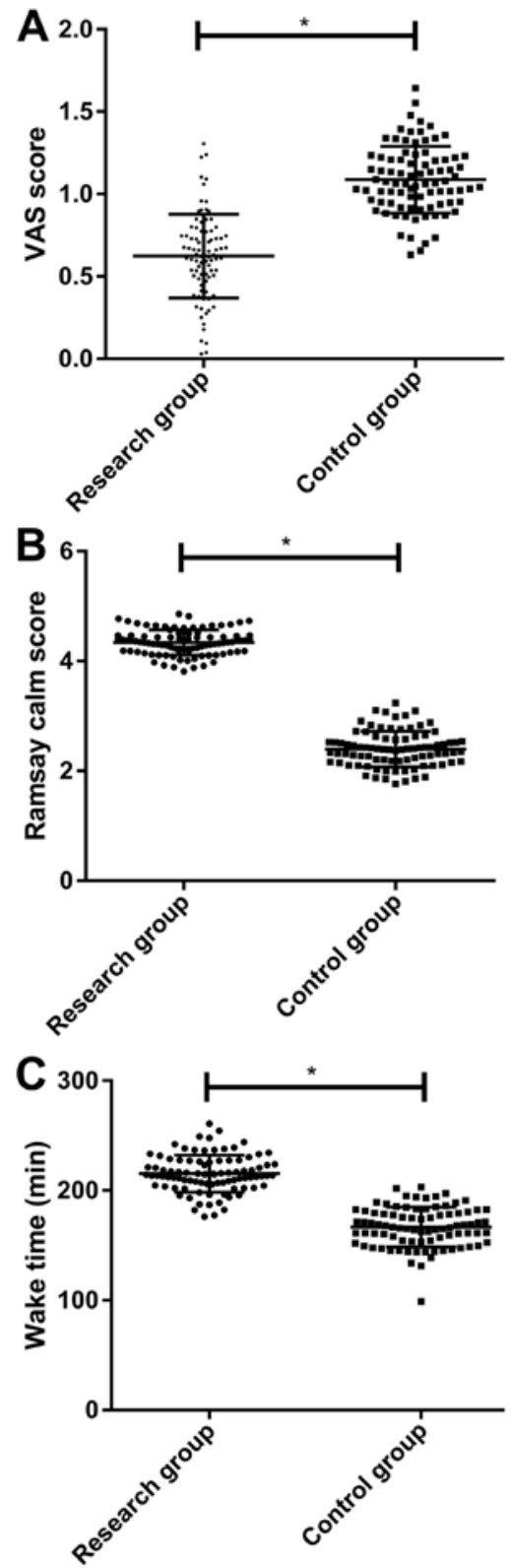

Figure 2. Comparison between the two groups in VAS score and Ramsay sedation scale score. (A) Comparison between the two groups in VAS score. (B) Comparison between the two groups in Ramsay sedation scale score. (C) Comparison between the two groups in anesthesia wake-up time. ${ }^{*} \mathrm{P}<0.001$. VAS, visual analogue scale.

and also showed no significant difference in Scr at the different time points. The research group showed a significantly lower level of BUN, RBP and Cys-C compared with the control group at days 1 and 3 after surgery $(\mathrm{P}<0.05$; Fig. 3$)$. Moreover, at days 1 and 3 after surgery, both groups showed no significant difference in Scr compare with levels before surgery, but showed significantly increased levels of BUN, RBP and Cys-C $(\mathrm{P}<0.05$; Fig. 3). Furthermore, it was found that 3 days after surgery, both groups showed significantly lower levels BUN, $\mathrm{RBP}$ and Cys-C compared with levels 1 day after surgery $(\mathrm{P}<0.05$; Fig. 3).

Comparison of inflammatory factors. Both groups showed no significant difference in the levels of TNF- $\alpha$, IL- 6 and IL- 8 before surgery (Fig. 4). It was found that the level of 

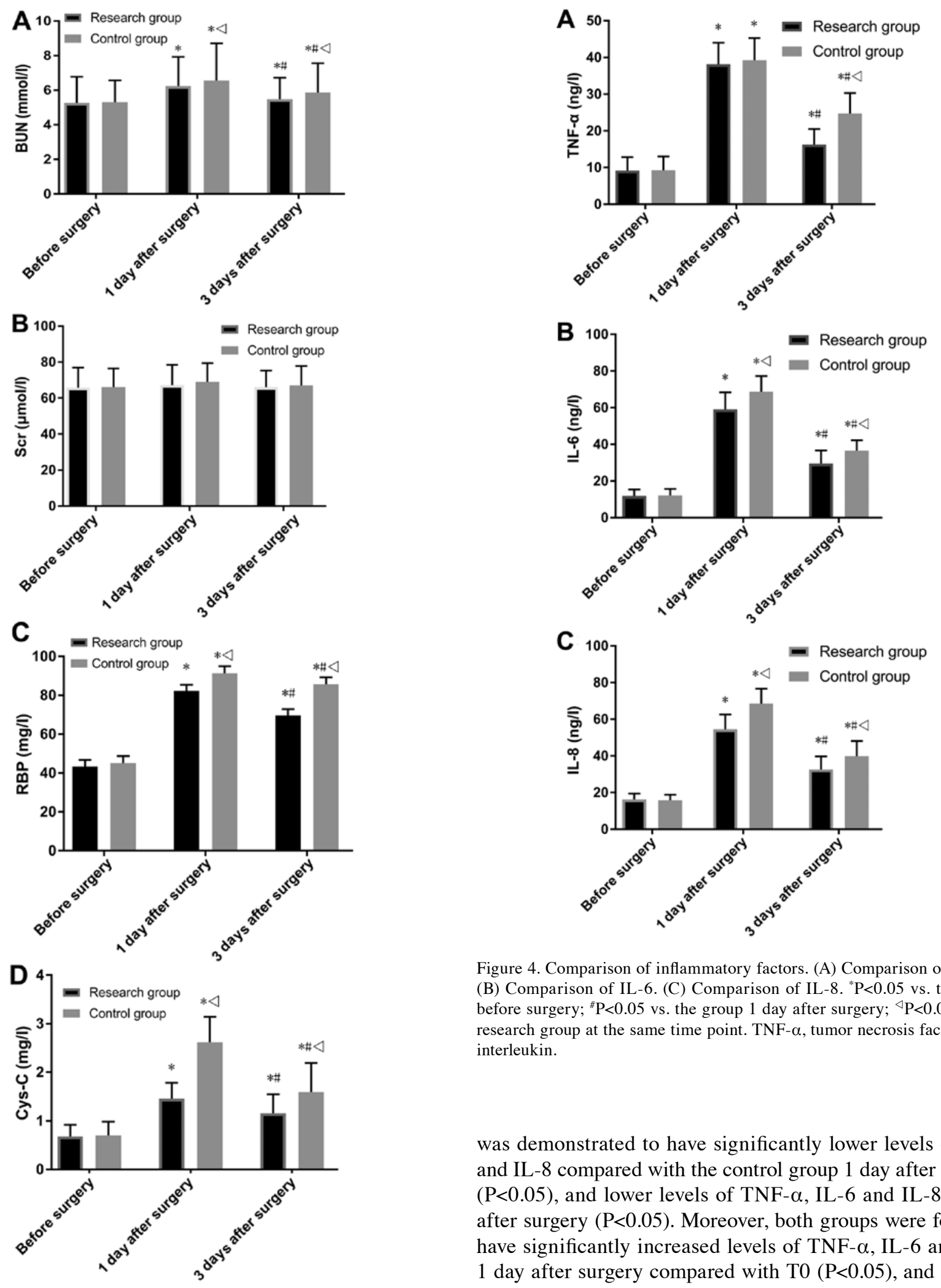

Figure 4. Comparison of inflammatory factors. (A) Comparison of TNF- $\alpha$. (B) Comparison of IL-6. (C) Comparison of IL-8. "P<0.05 vs. the group before surgery; ${ }^{\prime} \mathrm{P}<0.05$ vs. the group 1 day after surgery; ${ }^{\triangleleft} \mathrm{P}<0.05$ vs. the research group at the same time point. TNF- $\alpha$, tumor necrosis factor $\alpha$; IL, interleukin.

Figure 3. Comparison of renal function. (A) Comparison of BUN. (B) Comparison of Scr. (C) Comparison of RBP. (D) Comparison in Cys-C. ${ }^{*} \mathrm{P}<0.001$ vs. the same group before surgery; ${ }^{\#} \mathrm{P}<0.001$ vs. the same group 1 day after surgery; ${ }^{\triangleleft} \mathrm{P}<0.001$ vs. the research group at the same time point. BUN, blood urea nitrogen; Scr, serum creatinine; RBP, retinol binding protein; Cys-C, cystatin C.

TNF- $\alpha$ was not significantly different in the research group compared with the control group. However, the research group

was demonstrated to have significantly lower levels of IL-6 and IL-8 compared with the control group 1 day after surgery $(\mathrm{P}<0.05)$, and lower levels of TNF- $\alpha$, IL-6 and IL-8 3 days after surgery $(\mathrm{P}<0.05)$. Moreover, both groups were found to have significantly increased levels of TNF- $\alpha$, IL- 6 and IL-8 1 day after surgery compared with $\mathrm{T} 0(\mathrm{P}<0.05)$, and showed lower levels 3 days after surgery compared with levels 1 day after surgery $(\mathrm{P}<0.05$; Fig. 4$)$.

Comparison of immune function. The present results suggested that the two groups showed no significant differences in the expression levels of $\mathrm{CD}^{+}, \mathrm{CD}^{+}, \mathrm{CD}^{+}, \mathrm{CD}^{+} / \mathrm{CD}^{+}$and $\mathrm{NK}$. However, 1 day after surgery, the research group had significantly higher expression levels of $\mathrm{CD}^{+}, \mathrm{CD}^{+}, \mathrm{CD}^{+}-\mathrm{CD} 8^{+}$ and NK cells, and significantly lower levels of $\mathrm{CD} 8^{+}$compared with the control group ( $\mathrm{P}<0.001$; Fig. 5). Moreover, 3 days 

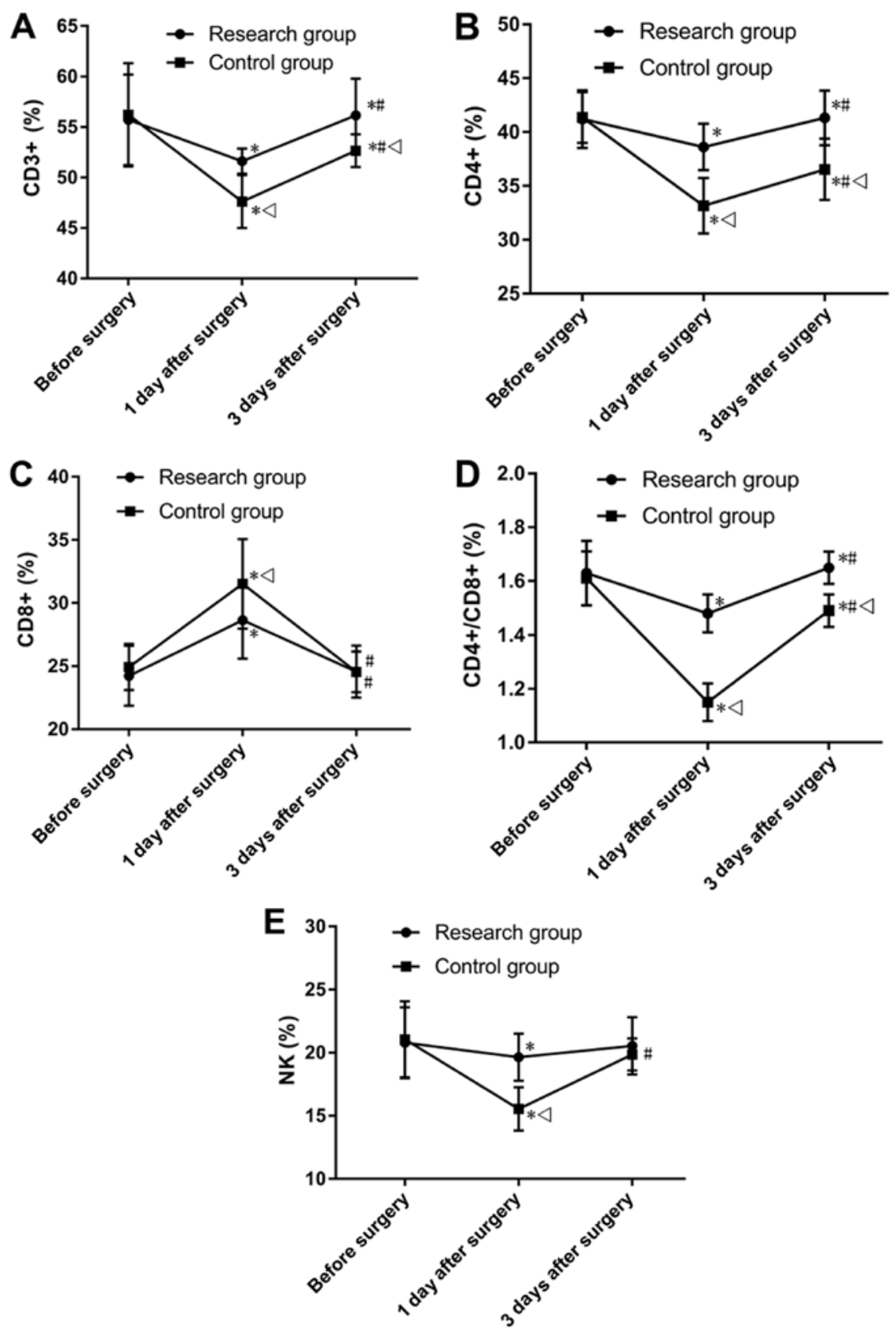

Figure 5. Comparison of immune function. (A) Comparison of $\mathrm{CD}^{+}$. (B) Comparison in $\mathrm{CD}^{+}$. (C) Comparison in $\mathrm{CD} 8^{+}$. (D) Comparison in $\mathrm{CD}^{+} / \mathrm{CD}^{+}$. (E) Comparison between the two groups in NK cells. ${ }^{*} \mathrm{P}<0.001$ vs. the group before surgery; ${ }^{*} \mathrm{P}<0.001$ vs. the group 1 day after surgery; ${ }^{\triangleleft} \mathrm{P}<0.001$ vs. the research group at the same time point. NK, natural killer.

after surgery, no significant difference was found between the two groups for the expression levels of $\mathrm{CD}^{+}$and NK cells, however the research group had significantly higher levels of $\mathrm{CD}^{+}, \mathrm{CD}^{+}$and $\mathrm{CD}^{+} / \mathrm{CD}^{+}$compared with the control group $(\mathrm{P}<0.001$; Fig. 5).

Comparisons of hospitalization time and hospitalization expense. The hospitalization time of the research group was significantly shorter compared with the control group (3.62 \pm 1.20 and 5.24 \pm 2.26 days, respectively; $\mathrm{P}<0.001$; Fig. 6). Furthermore, the present results suggested that there was no significant difference between the research group and control group in hospitalization expense (4563.62 \pm 315.64 and 4602.56 \pm 275.64 CNY, respectively).

\section{Discussion}

Percutaneous nephrolithotomy is a minimally invasive surgery associated with a small amount of intraoperative blood loss and fast recovery. However, during percutaneous nephrolithotomy, high pressure pouring is required, which can increase renal pelvic pressure in patients and lead to urine countercurrent in renal pelvis (21). Therefore, the correct anesthetic drug for percutaneous nephrolithotomy is needed to avoid renal damage. In order reduce the damage on renal function of patients with kidney calculi during operation, several studies have investigated and confirmed the role of different anesthetics in protecting renal function $(22,23)$. 


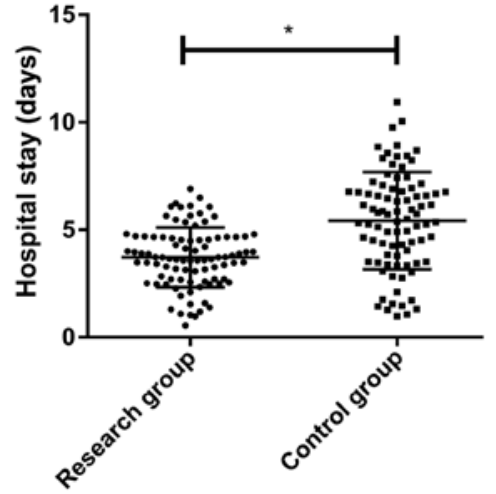

Figure 6. Comparison of the hospitalization time between the two groups. ${ }^{*} \mathrm{P}<0.001$.

Dexmedetomidine, an imidazole derivative, has a very high selective binding with $\alpha 2$ adrenergic receptor and acts as a $\alpha 2$ adrenergic agonist, with high efficiency and high selectivity. The binding products of dexmedetomidine not only inhibit the sympathetic nervous system, but also can reduce the release of norepinephrine, which helps provide protection to the kidneys (24). Ammar et al (25) demonstrated that dexmedetomidine has a protective effect on kidney function in cardiac surgery.

The present results suggested that the vital sign parameters of the research group were lower compared with the control group after anesthesia, suggesting that dexmedetomidine may effectively reduce the fluctuation of blood flow parameters during the perioperative period of percutaneous nephrolithotomy. Furthermore, it was found that $\mathrm{SpO}_{2}$ was not significantly different between the two groups at different time points, suggesting that dexmedetomidine did not inhibit the respiratory function and blood circulation of patients. The present results were consistent with previous results from Liu et al (26), which demonstrated the effects of dexmedetomidine on the microcirculation of patients during cardiac surgery. Moreover, it was found that the VAS and Ramsay sedation scale scores of the research group were different from those of the control group after surgery, suggesting that dexmedetomidine may be an effective sedative and analgesic agent for patients during the perioperative period. The research group experienced longer anesthesia wake-up time compared with the control group, which may be due to the fact that dexmedetomidine plays a synergistic role in enhancing the intensity of local anesthetic drugs and prolongs the action time of drugs (27). In addition, patients in the control group relied on intravenous injection for maintenance of intraoperative anesthesia, as this group did not receive dexmedetomidine anesthesia and intravenous anesthesia was stopped when the surgery ended.

A previous study has confirmed that conventional anesthesia is not as effective as dexmedetomidine in surgical operations (28). The present results suggested that there were no significant differences between the two groups for the incidence of adverse reactions, suggesting that dexmedetomidine had a relatively high safety profile in percutaneous nephrolithotomy. However, Jessen Lundorf et al (29) found that patients treated with dexmedetomidine for analgesia were more likely to have hypotension, while the incidence of hypotension in the present study was only $2.20 \%$ (two patients). This inconsistency may be because the anesthetics used by Jessen Lundorf et al (29) and the present study were not identical, so the difference may be related to a specific reaction from dexmedetomidine combined with other anesthetics, which increases the likelihood of hypotension in patients. Furthermore, the difference in results may be because the surgery performed by Jessen Lundorf et al (29) was an abdominal surgery with relatively large trauma, while percutaneous nephrolithotomy is a minimally invasive surgery with a small amount of intraoperative blood loss. The hemodynamics of patients undergoing percutaneous nephrolithotomy can be maintained at a relatively stable state, so the possibility of decreased blood pressure was low. However, further studies are required to investigate the safety of dexmedetomidine for percutaneous nephrolithotomy.

The renal function, inflammatory factors, and immune function were compared between the two groups, and the results indicated that that the renal function and immune function of the research group were both improved compared with the control group, while the levels of inflammatory factors in the research group were lower compared with the control group. As routine conventional indexes of renal injury, BUN and Scr levels were not significantly different in the present study, which may be due to the fact that the renal injury caused by percutaneous nephrolithotomy was small. It has been previously reported that BUN and Scr show a significant increase when the renal injury reaches a relatively severe state (30). Thus, further studies are required to investigate this effect. As a good index of early renal injury, Cys-C can be completely reabsorbed by the proximal convoluted tubule after being filtered by the renal tubular (31), and RBP is a sensitive index for the degree of injury of the proximal convoluted tubule (32). In the present study, Cys-C and RBP levels were increased after surgery in the two groups, while those of the research group were lower compared with the control group, which suggested the protective effect of dexmedetomidine on renal function.

Due to limited experimental conditions, the present study was not able to investigate the protective mechanism of dexmedetomidine on renal function, which is a limitation. The present study hypothesized that the protection mechanism of dexmedetomidine may be as follows: Dexmedetomidine mainly acted in the sympatho-adrenomedullary system and did not bind to cytokines in peripheral blood to increase the concentration of catecholamine (33), which caused dilatation of small arteries in the renal tubular and relieved the pressure in renal capsule to maintain a relatively stable state of renal function. The present results suggested that the research group had lower levels of TNF- $\alpha$, IL-6 and IL-8 compared with the control group, suggesting that dexmedetomidine also had a beneficial anti-inflammatory effect. Previous results by Luo et al (34) and $\mathrm{Li}$ and Liu (35) are in line with results from the present study. Therefore, the protective effect of dexmedetomidine on renal function may also be related to its anti-inflammatory effect. Thus, dexmedetomidine may protect the kidney by reducing the inflammatory response in the body and lowering the possibility of oxidative stress in patients.

The present study also compared $\mathrm{T}$ lymphocyte subsets in the two groups and found that all patients showed decreased 
levels of $\mathrm{CD}^{+}, \mathrm{CD}^{+}, \mathrm{CD}^{+} / \mathrm{CD}^{+}$and $\mathrm{NK}$ and increased $\mathrm{CD}^{+}$, but that these cells gradually recovered 3 days after surgery. A previous study has shown that anesthetics cause changes in the immune function of patients at any duration of administration (36), which was also verified by the results of the present study. It was also demonstrated that the immune function of the patient was inhibited after surgery and anesthesia, but returned to the original level with the recovery of the patient. However, the research group had increased changes in immune function compared with the control, which suggested that dexmedetomidine also had a protective effect on the immune function. At present, there are few studies on the effects of dexmedetomidine on human immune function and the underlying mechanisms are not fully understood. Based on the present results, dexmedetomidine may reduce the immunosuppressive stress response on body by inhibiting sympathetic activation, or may reduce immunosuppression caused by the inflammatory response by downregulating inflammatory factors. Moreover, a previous study has shown that opiate drugs, such as propofol, have a more significant inhibitory effect on immune function compared with other opiate drugs (37). Furthermore, Chen et al (38) revealed that dexmedetomidine can inhibit the maturation and function of dendritic cells of human cord blood by impacting the synthesis of IL-12 and IL-23, and also has a regulatory effect on the normal operation of the immune function. Thus, this may be why the research group showed higher levels of $\mathrm{CD}^{+}$and $\mathrm{CD}^{+}$, and lower levels of $\mathrm{CD}^{+}$compared with the control group in the present study. However, due to the absence of in vitro experiments, it is difficult to determine the underlying mechanisms, thus additional studies are required. The present results suggested that the research group had a significantly improved rehabilitation process compared with the control group and that there was no difference in the hospitalization expense between the two groups, which indicated that it may be feasible to use dexmedetomidine in clinical practice.

The present study had some limitations. Experiments were not performed to determine the mechanism of dexmedetomidine on inflammatory indexes. In addition, due to the limited number of patients, homogenous ethnicity and small age range of patients, it is not possible to determine if the difference in performance of dexmedetomidine may be caused by changes in body functions. Therefore, further experiments are required, with in-depth analysis and discussion of the application of dexmedetomidine in percutaneous nephrolithotomy.

In conclusion, with relatively high safety profile, dexmedetomidine for sedation can effectively protect renal and immune functions and reduce the inflammatory response of patients during percutaneous nephrolithotomy, and thus may be used for application in clinical practice.

\section{Acknowledgements}

Not applicable.

\section{Funding}

No funding was received.

\section{Availability of data and materials}

The datasets used and/or analyzed during the present study are available from the corresponding author on reasonable request.

\section{Authors' contributions}

YB conceived the study and wrote the manuscript. HH was responsible for the ELISA. PZ analyzed and interpreted the patient data. WL and LH helped with statistical analysis. All authors read and approved the final manuscript.

\section{Ethics approval and consent to participate}

The study was approved by the Ethics Committee of The Second Affiliated Hospital of Fujian Medical University. Patients who participated in this research, signed the informed consent and had complete clinical data.

\section{Patient consent for publication}

Not applicable.

\section{Competing interests}

The authors declare that they have no competing interests.

\section{References}

1. Alatab S, Pourmand G, El Howairis MelF, Buchholz N, Najafi I, Pourmand MR, Mashhadi R and Pourmand N: National profiles of urinary calculi: A comparison between developing and developed worlds. Iran J Kidney Dis 10: 51-61, 2016

2. Türk C, Petř́́k A, Sarica K, Seitz C, Skolarikos A, Straub M and Knoll T: EAU Guidelines on Interventional Treatment for Urolithiasis. Eur Urol 69: 475-482, 2016.

3. Assimos D, Krambeck A, Miller NL, Monga M, Murad MH, Nelson CP, Pace KT, Pais VM Jr, Pearle MS, Preminger GM, et al: Surgical management of stones:american urological association/endourological society guideline, Part I. J Urol 196: 1153-1160, 2016.

4. Daudon M, Jungers P, Bazin D and Williams JC Jr: Recurrence rates of urinary calculi according to stone composition and morphology. Urolithiasis 46: 459-470, 2018.

5. Khan SR, Pearle MS, Robertson WG, Gambaro G, Canales BK, Doizi S, Traxer O and Tiselius HG: Kidney stones. Nat Rev Dis Primers 2: 16008, 2016.

6. Romero V, Akpinar H and Assimos DG: Kidney stones: A global picture of prevalence, incidence, and associated risk factors. Rev Urol 12: e86-e96, 2010.

7. Heers H and Turney BW: Trends in urological stone disease: A 5-year update of hospital episode statistics. BJU Int 118: 785-789, 2016.

8. Sofia NH and Walter TM: Prevalence and risk factors of kidney stone. GJRA 5: 183-187, 2016.

9. Abeywickarama B, Ralapanawa $U$ and Chandrajith R: Geoenvironmental factors related to high incidence of human urinary calculi (kidney stones) in Central Highlands of Sri Lanka. Environ Geochem Health 38: 1203-1214, 2016.

10. Ghani KR, Andonian S, Bultitude M, Desai M, Giusti G, Okhunov Z, Preminger GM and de la Rosette J: Preminger GMand de la Rosette J: Percutaneous nephrolithotomy:update, trends, and future directions. Eur Urol 70: 382-396, 2016.

11. Gorbachinsky I, Wood K, Colaco M, Hemal S, Mettu J, Mirzazadeh M, Assimos DG and Gutierrez-Aćeves J: Evaluation of renal function after percutaneous nephrolithotomy-does the number of percutaneous access tracts matter? J Urol 196: 131-136, 2016. 
12. Daggülli M, Utangac MM, Dede O, Bodakci MN, Hatipoglu NK, Penbegül N, Sancaktutar AA, Bozkurt Y and Söylemez H: Potential biomarkers for the early detection of acute kidney injury after percutaneous nephrolithotripsy. Ren Fail 38: 151-156, 2016.

13. Weerink MAS, Struys MMRF, Hannivoort LN, Barends CRM, Absalom AR and Colin P: Clinical pharmacokinetics and pharmacodynamics of dexmedetomidine. Clin Pharmacokinet 56: 893-913, 2017.

14. Edvardsson VO, Goldfarb DS, Lieske JC, Beara-Lasic L, Anglani F, Milliner DS and Palsson R: Hereditary causes of kidney stones and chronic kidney disease. Pediatr Nephrol 28: 1923-1942, 2013.

15. Doyle DJ, Goyal A, Bansal P, Garmon EH. American Society of Anesthesiologists Classification. 2020 Jul 4. In: StatPearls [Internet]. Treasure Island (FL): StatPearls Publishing; 2020.

16. Gurbet A, Basagan-Mogol E, Turker G, Ugun F, Kaya FN and Ozcan B: Intraoperative infusion of dexmedetomidine reduces perioperative analgesic requirements. Can J Anaesth 53: 646-652, 2006.

17. Lai HC, Chan SM, Lu CH, Wong CS, Cherng CH and Wu ZF: Planning for operating room efficiency and faster anesthesia wake-up time in open major upper abdominal surgery. Medicine (Baltimore) 96: e6148, 2017.

18. Cline ME, Herman J, Shaw ER and Morton RD: Standardization of the visual analogue scale. Nurs Res 41: 378-380, 1992.

19. Dawson R, von Fintel N and Nairn S: Sedation assessment using the Ramsay scale. Emerg Nurse 18: 18-20, 2010.

20. Bhowmick D, Sheridan RTC, Bushnell TP, Spalding KL. Practical Guidelines for Optimization and Characterization of the Beckman Coulter CytoFLEX ${ }^{\mathrm{TM}}$ Platform. Cytometry A 97(8):800-810, 2020

21. Ozayar E, Gulec H, Bayraktaroglu M, Tutal ZB, Kurtay A, Babayigit M, Ozayar A and Horasanli E: Comparison of retrograde intrarenal surgery and percutaneous nephrolithotomy: From the view of an anesthesiologist. J Endourol 30: 184-188, 2016.

22. Motayagheni N, Phan S, Eshraghi C, Nozari A and Atala A: A review of anesthetic effects on renal function: Potential organ protection. Am J Nephrol 46: 380-389, 2017.

23. Kim N, Lee JG, Lee S, Nam KS, Shu JW, Paik HC and Yoo YC: A comparison of propofol based total intravenous anesthesia and sevoflurane based balanced anesthesia on renal protection during lung transplantation under extracorporeal membrane oxygenation-A prospective, randomized trial. J Heart Lung Transplant 36: S116-S117, 2017.

24. Palomino J, Echavarria R, Franco-Acevedo A, MorenoCarranza B and Melo Z: Opioids preconditioning upon renal function and ischemia-reperfusion injury: A Narrative Review. Medicina (Kaunas) 55: 522, 2019.

25. Ammar AS, Mahmoud KM, Kasemy ZA and Helwa MA Cardiac and renal protective effects of dexmedetomidine in cardiac surgeries: A randomized controlled trial. Saudi J Anaesth 10: 395-401, 2016.

26. Liu X, Zhang K, Wang W, Xie G, Cheng B, Wang Y, Hu Y and Fang X: Dexmedetomidine versus propofol sedation improves sublingual microcirculation after cardiac surgery: A randomized controlled trial. J Cardiothorac Vasc Anesth 30: 1509-1515, 2016.
27. Zhang W, Xu W, Ning C, Li M, Zhao G, Jiang W, Ding J and Chen X: Long-acting hydrogel/microsphere composite sequentially releases dexmedetomidine and bupivacaine for prolonged synergistic analgesia. Biomaterials 181: 378-391, 2018.

28. Wang G, Niu J, Li Z, Lv H and Cai H: The efficacy and safety of dexmedetomidine in cardiac surgery patients: A systematic review and meta-analysis. PLoS One 13: e0202620, 2018.

29. Jessen Lundorf L, Korvenius Nedergaard H and Møller AM: Perioperative dexmedetomidine for acute pain after abdominal surgery in adults. Cochrane Database Syst Rev 2: CD010358, 2016.

30. Chan WW, Waltman Johnson K, Friedman HS and Navaratnam P: Association between cardiac, renal, and hepatic biomarkers and outcomes in patients with acute heart failure. Hosp Pract 1995 44: 138-145, 2016.

31. Zhang M, Li Y, Yang X, Shan H, Zhang Q, Ming Z, Xie Y, Chen H, Liu Y and Zhang J: Serum cystatin $C$ as an inflammatory marker in exacerbated and convalescent COPD patients. Inflammation 39: 625-631, 2016.

32. Nielsen R, Christensen EI and Birn H: Megalin and cubilin in proximal tubule protein reabsorption: From experimental models to human disease. Kidney Int 89: 58-67, 2016.

33. Sichrovsky TC, Mittal S and Steinberg JS: Dexmedetomidine sedation leading to refractory cardiogenic shock. Anesth Analg 106: $1784-1786,2008$

34. Luo X, Zheng X and Huang H: Protective effects of dexmedetomidine on brain function of glioma patients undergoing craniotomy resection and its underlying mechanism. Clin Neurol Neurosurg 146: 105-108, 2016.

35. Li Y and Liu S: The effect of dexmedetomidine on oxidative stress response following cerebral ischemia-reperfusion in rats and the expression of intracellular adhesion molecule-1 (ICAM-1) and S100B. Med Sci Monit 23: 867-873, 2017.

36. Xu Q, Shi NJ, Zhang $\mathrm{H}$ and Zhu YM: Effects of combined general-epidural anesthesia and total intravenous anesthesia on cellular immunity and prognosis in patients with non-small cell lung cancer: A comparative study. Mol Med Rep 16: 4445-4454, 2017.

37. Liu W, Wu L, Zhang M and Zhao L: Effects of general anesthesia with combined epidural anesthesia on inflammatory response in patients with early-stage gastric cancer undergoing tumor resection. Exp Ther Med 17: 35-40, 2019.

38. Chen G, Le Y, Zhou L, Gong L, Li X, Li Y, Liao Q, Duan K, Tong $\mathrm{J}$ and Ouyang W: Dexmedetomidine inhibits maturation and function of human cord blood-derived dendritic cells by interfering with synthesis and secretion of IL-12 and IL-23. PLoS One 11: e0153288, 2016.

This work is licensed under a Creative Commons Attribution-NonCommercial-NoDerivatives 4.0 International (CC BY-NC-ND 4.0) License. 